\title{
Mathematical modeling of a co-extrusion process for preparing gradient-index polymer optical fibers
}

\author{
Bo-Tau Liu, Wen-Chang Chen, Jyh-Ping Hsu* \\ Department of Chemical Engineering, National Taiwan University, Taipei 10617, Taiwan, ROC
}

Received 28 July 1997; revised 30 March 1998; accepted 13 May 1998

\begin{abstract}
The behavior of a closed co-extrusion process for the preparation of gradient-index polymer optical fibers is modeled theoretically. In particular, the effects of the essential parameters such as the volume ratios of the inner/outer layer, diffusivities of monomers, diffusion length, and monomer concentrations, on the distribution of refractive index (RI) are examined. The applicability of the model derived is justified by comparing with the experimental results from the literature. The latter are gathered from a system using poly(methyl methacrylate) as the host polymer, and methyl methacrylate and benzyl methacrylate as monomers. Our results show that the RI distribution of the fibers prepared by the co-extrusion process can be significantly adjusted by the essential parameters. (C) 1998 Elsevier Science Ltd. All rights reserved.
\end{abstract}

Keywords: Co-extrusion process; Mathematical modeling; PBzMA-PMMA optical fiber

\section{Introduction}

Owing to its versatile applications in optical fiber communication and imaging, gradient-index (GRIN) polymer has become a very attractive material in recent years [1-5]. Various GRIN polymer optical fibers were prepared successfully on a laboratory scale. Ishigure et al. [6], for example, produced a high bandwidth GRIN polymer optical fiber, which exhibits gigabit signal transmission over $100 \mathrm{~m}$ using a $650 \mathrm{~nm}$ high speed laser diode. A GRIN polymer optical fiber amplifier with a high gain in the visible region was prepared successfully by Tagaya et al. [7] through doping Rhodamine B dye into the core of the fiber. GRIN polymer lenses made from polymer fibers have been used as the essential components of the Selfoc lens array (SLA) for fax machines [8]. One of the specific characteristics of a GRIN polymer is that it has a parabolic refractive index (RI) distribution. This is different from the conventional optical materials, which usually have a stepwise variation in RI.

One of the successful processes for the preparation of GRIN polymer fibers is the extrusion process, which comprises an internal diffusion and surface evaporation (IDSE) operation [8-12], and a multi-layer co-extrusion procedure [13-17]. A closed co-extrusion process was developed by

\footnotetext{
* Corresponding author. Fax: (+886) 2 23623040; e-mail:
} t8504009@ccms.ntu.edu.tw us to prepare GRIN polymer fibers recently [15-17]. We showed that this new process is capable of overcoming several major drawbacks in the conventional extrusion processes, e.g. poor reproducibility and low production rate. The RI profile of the GRIN polymer fiber prepared was found to be affected by the nature of the reactant mixtures, e.g. types of monomer and host polymer used and their compositions, and the operating conditions, e.g. the feeding speeds of mixtures, diffusivities of monomers, temperature, and the length of enclosed (diffusion) zone. Apparently, a mathematical model which is able to describe the quantitative behavior of the system under consideration is highly desirable, not only for the understanding of the effects of various parameters on the performance of the process, but also for the design of laboratory-scaled experiments.

In this study, a mathematical model for the closed coextrusion process developed previously [15-17] is derived. In particular, the RI profile of a GRIN polymer fiber is modeled quantitatively. The effects of the essential parameters, such as the volume ratios of the inner/outer layer, the diffusivities of monomers, diffusion length, and monomer concentration, on the distribution of RI are simulated.

\section{Experimental}

By referring to Fig. 1, reactant 1, which contained the solution of polymer PMMA and monomers MMA and 


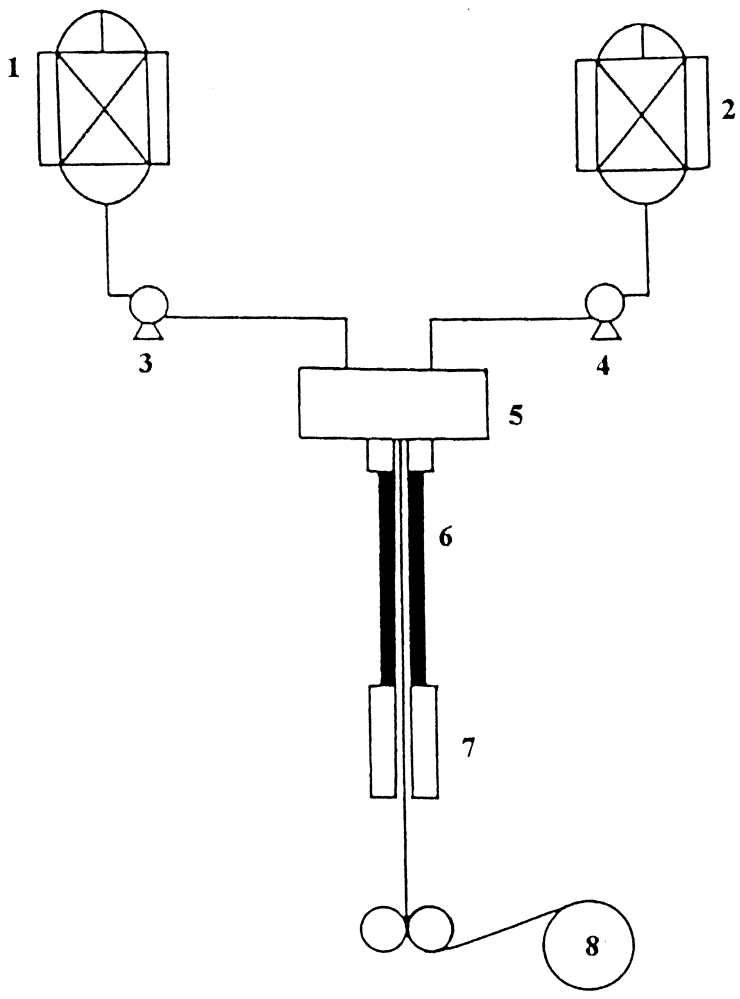

Fig. 1. Schematic representation of the co-extrusion process considered. 1 and 2: material supply tanks; 3 and 4: gear pumps; 5: concentric die; 6: enclosed (diffusion) zone; 7: hardening zone; 8: take-up roll.

BzMA, was placed in material supply tank 1. Similarly, reactant 2, which contained the solution of polymer PMMA and monomer MMA, was placed in material supply tank 2. These solutions were heated to $60^{\circ} \mathrm{C}$. The RIs of PMMA and PBzMA are 1.490 and 1.568 respectively. Gear pumps 3 and $\mathbf{4}$ were used to feed reactants 1 (inner layer) and 2 (outer layer) into a concentric die with

Table 1

Two sets of experiment conditions for comparing with the mathematical results

\begin{tabular}{lcc}
\hline Item & $\mathrm{a}^{\mathrm{a}}$ & $\mathrm{b}^{\mathrm{b}}$ \\
\hline Mixture 1 (inner layer) & & \\
PMMA (\%) & 58 & 58 \\
BzMA (\%) & 28 & 28 \\
MMA (\%) & 14 & 14 \\
& & \\
Mixture 2 (outer layer) & & \\
PMMA (\%) & 60 & 60 \\
BzMA (\%) & 0 & 0 \\
MMA (\%) & 40 & 40 \\
& & \\
$T\left({ }^{\circ} \mathrm{C}\right)$ & 80 & 90 \\
$R_{\mathrm{f}}(\mathrm{mm})$ & 0.25 & 0.42 \\
$R(\mathrm{~mm})$ & 0.5 & 0.83 \\
$u\left(\mathrm{~cm} \mathrm{~s}{ }^{-1}\right.$ ) & 4.244 & 4.244 \\
$L(\mathrm{~cm})$ & 45 & 45 \\
\hline
\end{tabular}

\footnotetext{
${ }^{\text {a }}$ Data from Ref. [15].
}

${ }^{\mathrm{b}}$ Data from Ref. [16].

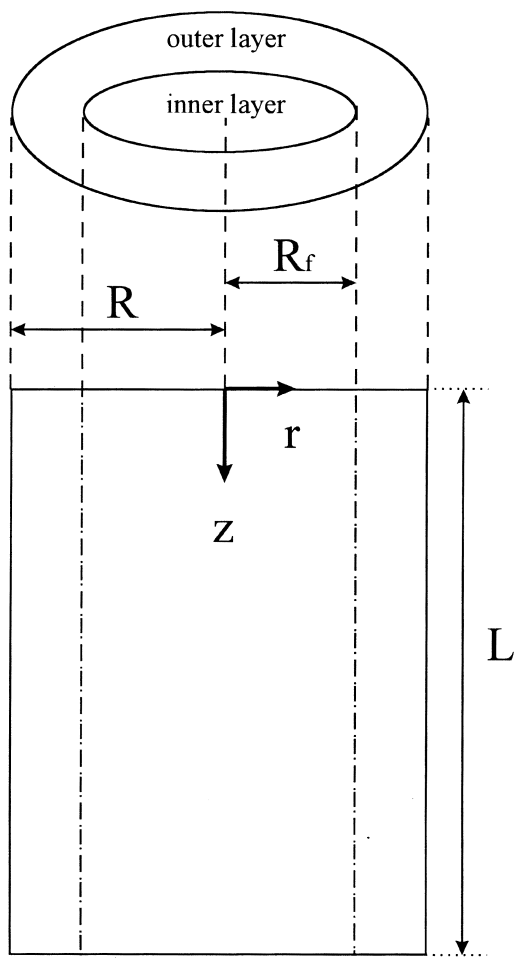

Fig. 2. Coordinates adopted in the mathematical modeling: $r$ and $z$ are the radial and axial distances respectively, $L$ is the length of diffusion zone, $R_{\mathrm{f}}$ and $R$ are the radii of the inner and outer layers of the diffusion zone respectively.

volumetric flow rates $0.5 \mathrm{~cm}^{3} \mathrm{~min}^{-1}$ and $1.5 \mathrm{~cm}^{3} \mathrm{~min}^{-1}$ respectively. A bi-layer composite filament was then extruded at a constant speed out of the orifice of the die $\mathbf{5}$ and fed into an enclosed (diffusion) zone $\mathbf{6}$, which was maintained at a constant temperature. Inside the diffusion zone, monomer BzMA diffused toward the outer layer, and monomer MMA toward the inner layer. This leads to a variation in the concentration profiles of monomers, and, therefore, the RI distribution in the filament. After passing the diffusion zone, the filament was fed to a hardening zone 7 where it was hardened by four UV lamps of $60 \mathrm{~W}$ each. A polymer fiber with a certain RI distribution is withdrawn by a take-up roll 8. Two sets of experimental conditions $[15,16]$ for comparing with the mathematical modeling results are shown in Table 1.

\section{Modeling}

A schematic representation of the system under consideration is shown in Fig. 2. Here, $R_{\mathrm{f}}$ denotes the radius of inner layer $R$ and $L$ the radius and the length of the diffusion zone respectively, $r$ the radial distance, and $z$ the distance measured from the top of the diffusion zone. Suppose that the operation is isothermal, and the diffusivities of BzMA and MMA are functions of the mass fraction of PMMA in the reactant mixtures only. Since the density of the filament remains approximately the same in the diffusion zone, we 
assume that the change in the bulk density of the filament is negligible. Suppose that MMA will not diffuse during the hardening period. In this case, the variation of the mass fraction $x$ of MMA or BzMA at a steady-state operation can be described by

$u \frac{\partial x}{\partial z}=D_{r}\left[\frac{\partial^{2} x}{\partial r^{2}}+\frac{1}{r} \frac{\partial x}{\partial r}\right]+D_{z} \frac{\partial^{2} x}{\partial z^{2}}$

where $u$ denotes the extrusion velocity, $t$ the time scale, and $D_{r}$ and $D_{z}$ the effective diffusivities of MMA or BzMA in the $r$ and $z$ directions respectively. The boundary conditions associated with Eq. (1) are assumed as follows:

$x$ is finite at $r=0$

$\frac{\partial x}{\partial r}=0$ at $r=R$

Eq. (2b) implies that the wall of the diffusion zone is impenetrable to MMA or BzMA. The inlet conditions for BzMA are

$x=x_{\mathrm{BzMAi}}, \quad 0<r<R_{r}$

$x=0, \quad R_{\mathrm{f}}<r<R$

where $x_{\mathrm{BzMAi}}$ is the inlet mass fraction of BzMA in the inner layer of the diffusion zone. The inlet conditions for MMA are

$x=x_{\mathrm{MMAi}}, \quad 0<r<R_{r}$

$x=x_{\text {MMAo }}, \quad R_{\mathrm{f}}<r<R$

where $x_{\mathrm{MMAi}}$ and $x_{\mathrm{MMAo}}$ are the inlet mass fractions of MMA in the inner and the outer layers of the diffusion zone respectively. For conditions of practical importance, the Peclect number $\left(u L / D_{z}\right)$ is large, i.e. the transport of MMA or BzMA due to convective motion is much significant than that due to molecular diffusion. Therefore, the last term on the righthand side of Eq. (1) is much smaller than the term on the left-hand side. In this case, Eq. (1) can be rewritten in the following dimensionless form:

$\frac{\partial x^{*}}{\partial z^{*}}=\frac{\partial^{2} x^{*}}{\partial r^{2}}+\frac{1}{r *} \frac{\partial x^{*}}{\partial r^{*}}$

where

$x *=\frac{x}{x_{0}}$

$z *=\frac{z D_{r}}{u R^{2}}$

$r *=\frac{r}{R}$

For BzMA, $x_{0}=x_{\mathrm{BzMAi}}$, and $x_{0}=x_{\mathrm{MMAo}}-x_{\mathrm{MMAi}} \equiv x_{\mathrm{MMAd}}$ for MMA. Since the diffusivity is assumed to remain constant, the solutes do not influence each other. The corresponding boundary and inlet conditions become

$x *$ is finite at $r * 0$

$\frac{\partial x^{*}}{\partial r *}=0$ at $r *=1$

For BzMA, we have

$x *=1, \quad 0<r *<R_{\mathrm{f}}^{*}$

$x *=0, \quad R_{\mathrm{f}}^{*}<r *<1$

and for MMA we have

$x *=x_{\mathrm{MMAi}} / x_{\mathrm{MMd}} \equiv x_{\mathrm{MMAi}}^{*}, \quad 0<r *<R_{f}^{*}$

$x *=x_{\mathrm{MMAo}} / x_{\mathrm{MMd}}, \quad R_{\mathrm{f}}^{*}<r *<1$

where $R_{\mathrm{f}}^{*}=R_{\mathrm{f}} / R$. Solving Eq. (3) subject to Eqs. (4a)-(4d) gives the spatial variation of BzMA:

$x *=R_{\mathrm{f}}^{* 2}+2 R_{\mathrm{f}}^{*} \sum_{m=1}^{\infty} \frac{J_{1}\left(\beta_{m} R_{\mathrm{f}}^{*}\right)}{\beta_{m} J_{0}^{2}\left(\beta_{m}\right)} \exp \left(-\beta_{m}^{2} z *\right) J_{0}\left(\beta_{m} r *\right)$

Similarly, solving Eq. (3) subject to Eqs. (4a), (4b), (4e), and (4f) yields the spatial variation of MMA:

$$
\begin{aligned}
x *= & \left(1-R_{\mathrm{f}}^{* 2}\right)-2 R_{\mathrm{f}}^{*} \sum_{m=1}^{\infty} \frac{J_{1}\left(\beta_{m} R_{\mathrm{f}}^{*}\right)}{\beta_{m} J_{0}^{2}\left(\beta_{m}\right)} \\
& \times \exp \left(-\beta_{m}^{2} z *\right) J_{0}\left(\beta_{m} r *\right)+x_{\mathrm{MMAi}}^{*}
\end{aligned}
$$

The dimensionless mass fractions of BzMA and MMA at the outlet of the diffusion zone, $x_{\mathrm{BzMA}}^{*}$ and $x_{\mathrm{MMA}}^{*}$ respectively, can be obtained from Eqs. (5) and (6) as

$$
\begin{aligned}
x_{\mathrm{BzMA}}^{*}= & R_{\mathrm{f}}^{* 2}+2 R_{\mathrm{f}}^{*} \sum_{m=1}^{\infty} \frac{J_{1}\left(\beta_{m} R_{\mathrm{f}}^{*}\right)}{\beta_{m} J_{0}^{2}\left(\beta_{m}\right)} \\
& \times \exp \left(-\beta_{m}^{2} z_{\mathrm{BzMA}}^{*}\right) J_{0}\left(\beta_{m} r *\right) \\
x_{\mathrm{MMA}}^{*}= & \left(1-R_{\mathrm{f}}^{* 2}\right)-2 R_{\mathrm{f}}^{*} \sum_{m=1}^{\infty} \frac{J_{1}\left(\beta_{m} R_{\mathrm{f}}^{*}\right)}{\beta_{m} J_{0}^{2}\left(\beta_{m}\right)} \\
& \times \exp \left(-\beta_{m}^{2} z_{\mathrm{MMA}}^{*}\right) J_{0}\left(\beta_{m} r *\right)+x_{\mathrm{MMAi}}^{*}
\end{aligned}
$$

where $J_{0}$ and $J_{1}$ are the Bessel functions of the first kind of orders 0 and 1 respectively, $\beta_{m}$ is the positive root of $J_{1}\left(\beta_{m}\right)=0$. The scaled variables are defined by

$$
\begin{aligned}
& z_{\mathrm{BzMA}}^{*}=\frac{L D_{\mathrm{BzMA}}}{u R^{2}} \\
& z_{\mathrm{MMA}}^{*}=\frac{L D_{\mathrm{MMA}}}{u R^{2}} \\
& x_{B z M A}^{*}=\frac{x_{\mathrm{BzMA}}}{x_{\mathrm{BzMAi}}} \\
& x_{\mathrm{MMA}}^{*}=\frac{x_{\mathrm{MMA}}}{x_{\mathrm{MMAd}}}
\end{aligned}
$$




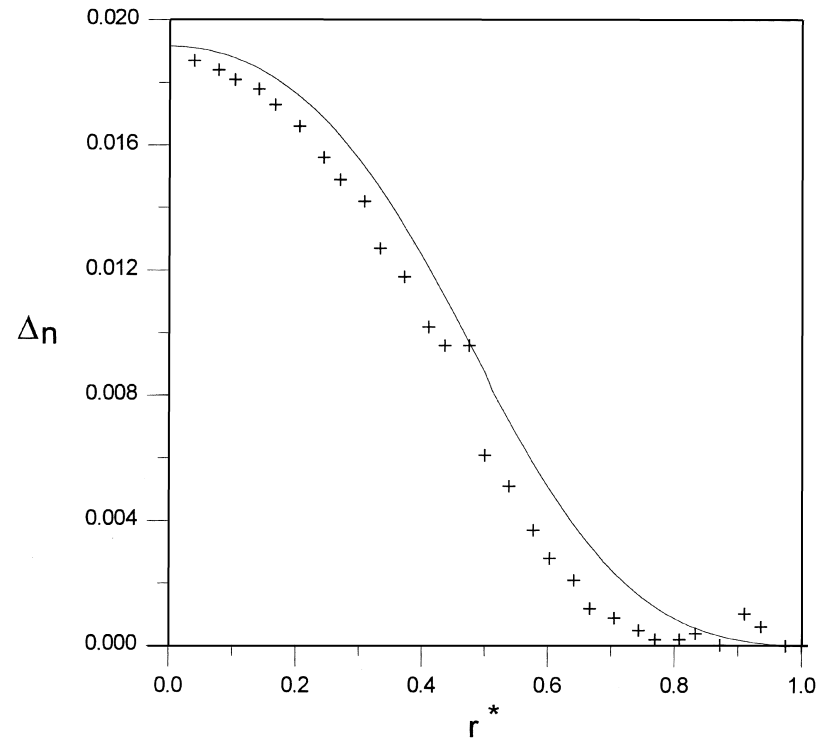

Fig. 3. Variation of $\Delta n$ as a function of $r^{*}$. The experimental conditions are shown in column (a) of Table 1 . The estimated values of the adjustable parameter are $D_{\mathrm{BzMA}}=4.72 \times 10^{-6} \mathrm{~cm}^{2} \mathrm{~s}^{-1}$ and $\mathrm{D}_{\mathrm{MMA}}=1.18 \times$ $10^{-5} \mathrm{~cm}^{2} \mathrm{~s}^{-1}$. $\times$ : experimental data; :- - values based on the present model.

Lorentz [18] and Lorenz [19] suggested that the optical property of a non-absorbing media and its chemical structure correlate through an additive rule. The RI $n_{\mathrm{D}}$ of a composite material, for example, can be represented by [20]

$n_{\mathrm{D}}=\sqrt{\frac{1+2 \phi}{1-\phi}}$

with

$\phi=\frac{\sum_{i} \frac{n_{\mathrm{D}, i}^{2}-1}{n_{\mathrm{D}, i}^{2}+2} \frac{x_{i}}{\rho_{i}}}{\sum_{i} \frac{x_{i}}{\rho_{i}}}$

where $n_{\mathrm{D}, i}$ and $\rho_{i}$ being, respectively, the RI and density of component $i$.

\section{Results and discussion}

\subsection{Justification of the mathematical modeling with the experimental results}

Figs 3 and 4 show the variation in the difference between the RI at the center of a fiber and that at a scaled radial distance from the center $r^{*}, \Delta n$, as a function of $r^{*}$. Both the data from the experimental conditions of Table 1 and the results predicted by the present model are shown in the figures. The comparison of the modeling data with the experimental data in these two figures shows a satisfactory agreement. This suggests that the present mathematical modeling can successfully predict the RI distribution of the GRIN polymer fibers from the closed co-extrusion

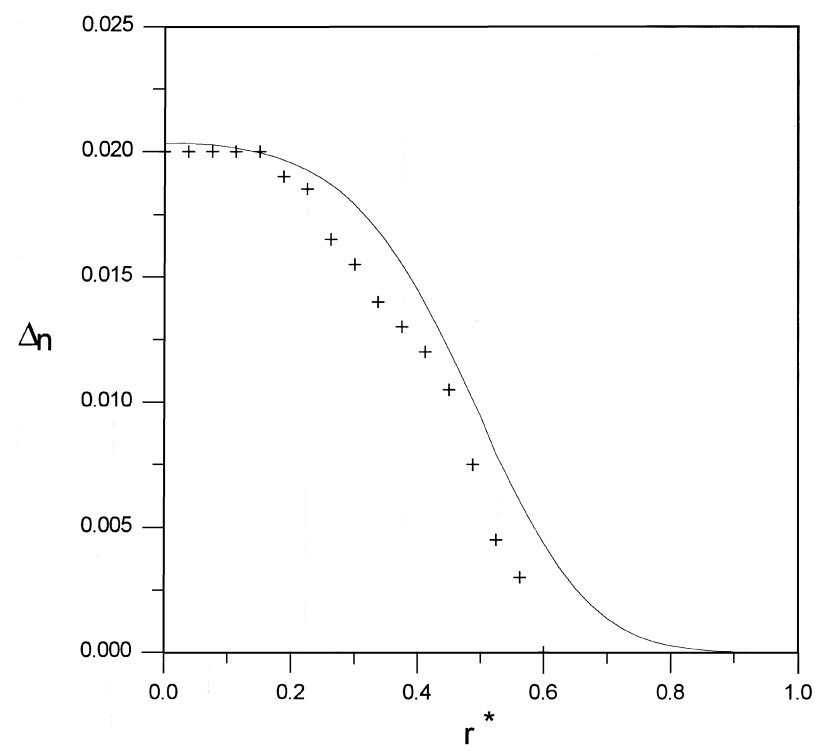

Fig. 4. Variation of $\Delta n$ as a function of $r^{*}$. The experimental conditions are shown in column (b) of Table 1 . The estimated values of the adjustable parameter are $D_{\mathrm{BzMA}}=6.55 \times 10^{-6} \mathrm{~cm}^{2} \mathrm{~s}^{-1}$ and $D_{\mathrm{MMA}}=2.62 \times$ $10^{-5} \mathrm{~cm}^{2} \mathrm{~s}^{-1}$. $\times$ : experimental data; - - -: present model.

process with [16] or without [15] the core-shell separation die design. Note that in Fig. 3, $\Delta n$ almost vanishes in the region near the boundary of the enclosed zone $\left(r^{*} \geq 0.75\right)$. This is due to the fact that the boundary is impenetrable to BzMA. However, the distribution of $\Delta n$ in the region of $0 \leq$ $r^{*} \leq 0.62$ (note: data from Ref. [15] by taking the linear portion of $\Delta n$ versus $r *$ $*^{2}$ ) is approximately parabolic. For the imaging application, the portion of the fiber corresponding to $r^{*} \geq 0.62$ will be cut off by a core-shell die. For the case of Fig. 4, the experimental data in the region $r^{*} \geq 0.6$ without the non-parabolic RI distribution are not shown. This is because the fiber portion corresponding to this region was cut off by the core-shell separation die design at the end of the diffusion zone. In the data fitting procedure, since the effective diffusivities of monomers $D_{\mathrm{BzMA}}$ and $D_{\mathrm{MMA}}$ are not available in the literature, they are treated as adjustable parameters. A least squares criterion is chosen for the estimation of $D_{\mathrm{BzMA}}$ and $D_{\mathrm{MMA}}$. The estimated values of $D_{\mathrm{BzMA}}$ and $D_{\mathrm{MMA}}$ are in the range of $10^{-5}-10^{-6} \mathrm{~cm}^{2} \mathrm{~s}^{-1}$, which are in the same order of magnitude as a small molecular ethyl acetate diffused in PMMA [21]. Note that, although both $D_{\mathrm{BzMA}}$ and $D_{\mathrm{MMA}}$ increase with the temperature, the ratio ( $\left.D_{\mathrm{MMA}} / D_{\mathrm{BzMM}}\right)$ decreases with the temperature. As will be discussed later, this ratio should also be considered in the design stage.

\subsection{Applications of the mathematical modeling to the co- extrusion process}

\subsubsection{Different volume ratios of the inner/outer layer, $R_{\mathrm{f}}^{*}$}

The simulated variations in $\Delta n$ as a function of $r *$ for various $R_{\mathrm{f}}^{*}$ (radius of inner layer/radius of diffusion zone) are illustrated in Fig. 5. This figure shows that, for a fixed $r^{*}$, 


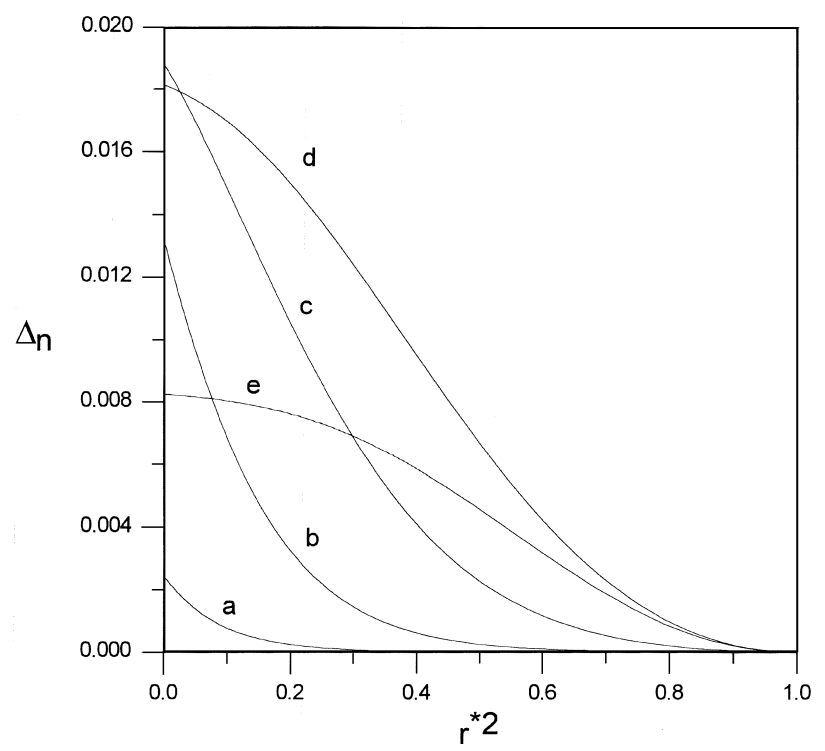

Fig. 5. Simulated variation of $\Delta n$ as a function of $r *^{2}$ at various $R_{\mathrm{f}}^{*}$ for the case $z_{\mathrm{BzMA}}^{*}=0.02$ and $z_{\mathrm{MMA}}^{*}=0.05$. The initial composition of a fiber is the same as that in Fig. 3. (a) $R_{\mathrm{f}}^{*}=0.1$; (b) $R_{\mathrm{f}}^{*}=0.3$; (c) $R_{\mathrm{f}}^{*}=0.5$; (d) $R_{\mathrm{f}}^{*}=0.7$; (e) $R_{\mathrm{f}}^{*}=0.9$.

$\Delta n$ increases with increasing $R_{\mathrm{f}}^{*}$, passes through a maximum (at $R_{\mathrm{f}}^{*}=0.7$ ), and then decreases with a further increase in $R_{\mathrm{f}}^{*}(=0.9)$. Also, the smaller the $R_{\mathrm{f}}^{*}$, the closer the anaclastic point of the RI curve to the center of a fiber. Note that if the distribution of RI is parabolic, i.e.

$n_{\mathrm{D}}(r *)=n_{\mathrm{D}}(r *=0)\left(1-\frac{A}{2} r *^{2}\right)$

where $A$ is constant, then a plot of $\Delta n$ versus $r *^{2}$ yields a straight line. Fig. 5 shows that, if $R_{\mathrm{f}}^{*}$ is too small or too large, the $\Delta n$ value and the portion of the parabolic RI distribution are too small to satisfy the requirements of imaging applications. This result can be explained as below. If $R_{\mathrm{f}}^{*}$ is too small, the fiber contains only a small portion of the high RI monomer BzMA. Also, it cannot diffuse too far to the outer layer. Thus, the $\Delta n$ value and the parabolic RI distribution will be too small in this case. On the other hand, if $R_{\mathrm{f}}^{*}$ is too large, the monomer BzMA will be uniformly distributed inside the fiber and results in the same kind of the RI distribution as the above case. In other words, if all the other conditions are fixed, there exists an optimum $R_{\mathrm{f}}^{*}$ which leads to a RI distribution most close to a parabolic distribution and a corresponding $\Delta n$ value as a GRIN polymer fiber for imaging applications.

\subsubsection{Different initial concentrations of the monomer BzMA}

The simulated variations of $\Delta n$ as a function of $r *$ for various initial concentrations of BzMA from 0.084 to the maximum value of 0.42 in the inner layer are presented in Fig. 6. The magnitude of $\Delta n$ is found to increase with increasing initial concentration of BzMA, since BzMA has a higher RI than MMA. This result is also confirmed by the experimental data of Ref. [16].

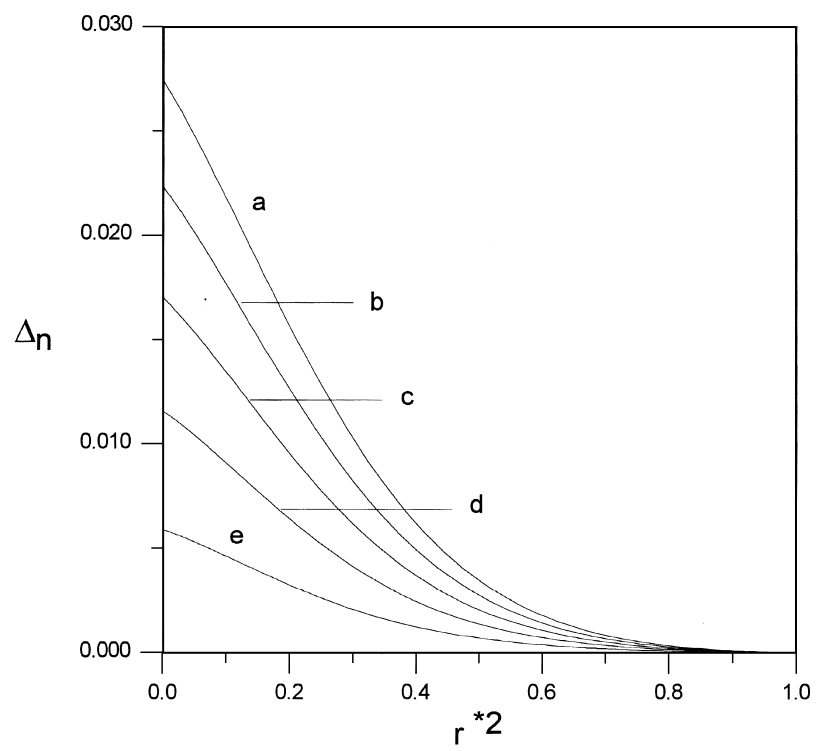

Fig. 6. Simulated variation of $\Delta n$ as a function of $r *^{2}$ at various initial concentrations of BzMA in the inner core for the case $R_{\mathrm{f}}^{*}=0.5$ $z_{\mathrm{BzMA}}^{*}=0.02$ and $z_{\mathrm{MMA}}^{*}=0.05$. Inner: 58\% PMMA; outer: $40 \%$ MMA and $60 \%$ PMMA. (a) $x_{\mathrm{BzMAi}}=0.420$; (b) $x_{\mathrm{BzMAi}}=0.336$; (c) $x_{\mathrm{BzMAi}}=0.252$; (d) $x_{\mathrm{BzMAi}}=0.168$; (e) $x_{\mathrm{BzMAi}}=0.084$.

\subsubsection{Different magnitudes of process parameters $z_{\mathrm{BzMA}}^{*}$ and $z_{\mathrm{MMA}}^{*}$}

Fig. 7 shows the simulated variation of $\Delta n$ as a function of $r *^{2}$ for various $z_{\mathrm{BzMA}}^{*}$ and $z_{\mathrm{MMA}}^{*}$. This figure suggests that a fiber with a larger $z_{\text {BzMA }}^{*}$ and $z_{\text {MMA }}^{*}$ is capable of having a larger region in which the distribution of RI is close to a parabolic distribution. The magnitude of $\Delta n$, however, decreases with the increase in both $z_{\mathrm{BzMA}}^{*}$ and $z_{\mathrm{MMA}}^{*}$. By

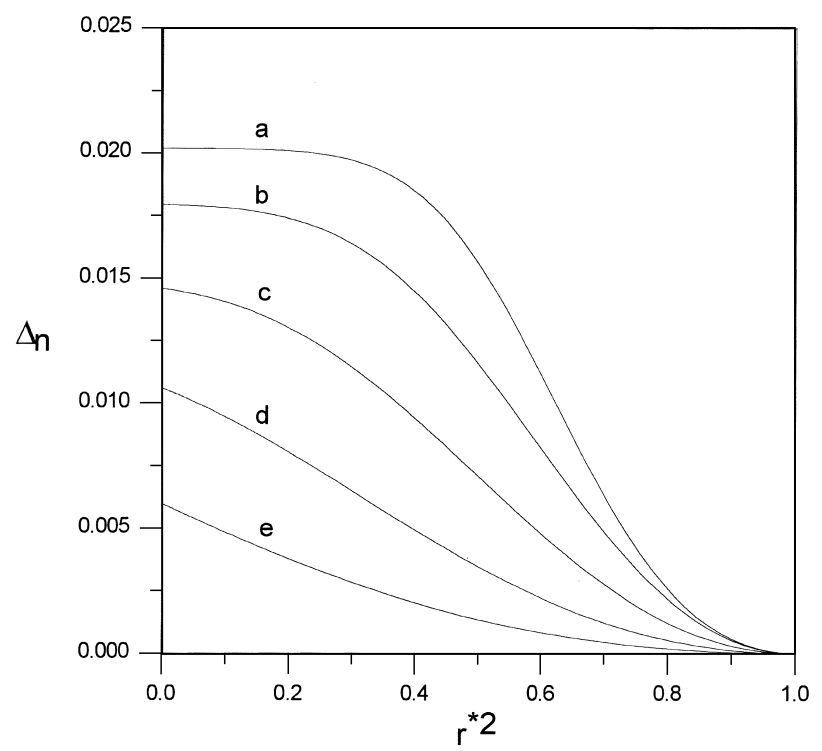

Fig. 7. Simulated variation of $\Delta n$ as a function of $r *^{2}$ at various values of $z_{\mathrm{BzMA}}^{*}$ and $z_{\mathrm{MMA}}^{*}$ for the case $R_{\mathrm{f}}^{*}=0.8, z_{\mathrm{BzMA}}^{*}=k \times 0.02$, and $z_{\mathrm{MMA}}^{*}=k \times 0.05, k$ being a proportional constant. Inner: $28 \%$ BzMA, 14\% MMA, and 58\% PMMA; outer: 40\% MMA and 60\% PMMA. (a) $k=0.25$; (b) $k=0.5$; (c) $k=1$; (d) $k=2$; (e) $k=4$. 


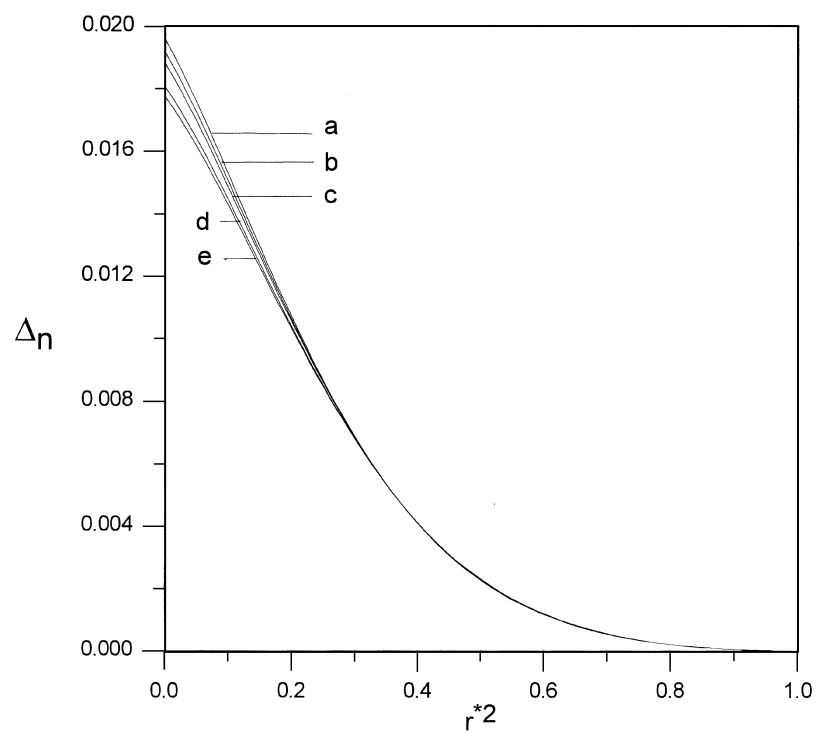

Fig. 8. Simulated variation of $\Delta n$ as a function of $r *^{2}$ at various values of $z_{\mathrm{MMA}}^{*}$ for the case $R_{\mathrm{f}}^{*}=0.5$ and $z_{\mathrm{BzMA}}^{*}=0.02$. Inner: $28 \%$ BzMA, 14\% MMA, and 58\% PMMA; outer: 40\% MMA and 60\% PMMA (a) $z_{\mathrm{MMA}}^{*}=0.03$; (b) $z_{\mathrm{MMA}}^{*}=0.04$; (c) $z_{\mathrm{MMA}}^{*}=0.05$; (d) $z_{\mathrm{MMA}}^{*}=0.08$; (e) $z_{\mathrm{MMA}}^{*}=0.1$.

referring to Eqs. (8a) and (8b), a large $z_{\mathrm{BzMA}}^{*}\left(z_{\mathrm{MMA}}^{*}\right)$ means a large monomer diffusion coefficients $D_{\mathrm{BzMA}}\left(D_{\mathrm{MMA}}\right)$, a small extrusion velocity $u$, a small fiber radius $R$, and/or a large diffusion length $L$. It results in a uniform distribution of both monomers BzMA and MMA in the fiber. Hence, a small $\Delta n$ value is formed with increasing $z_{\mathrm{BzMA}}^{*}$ and $z_{\mathrm{MMA}}^{*}$. Note that the qualitative behavior of the distribution of $\Delta n$ near the center of a fiber varies with $z_{\mathrm{BzMA}}^{*}$ and $z_{\mathrm{MMA}}^{*}$.

The simulated variation of $\Delta n$ as a function of $r * *^{2}$ for various $z_{\mathrm{MMA}}^{*}$ at a fixed $z_{\mathrm{BzMA}}^{*}$ is illustrated in Fig. 8 .

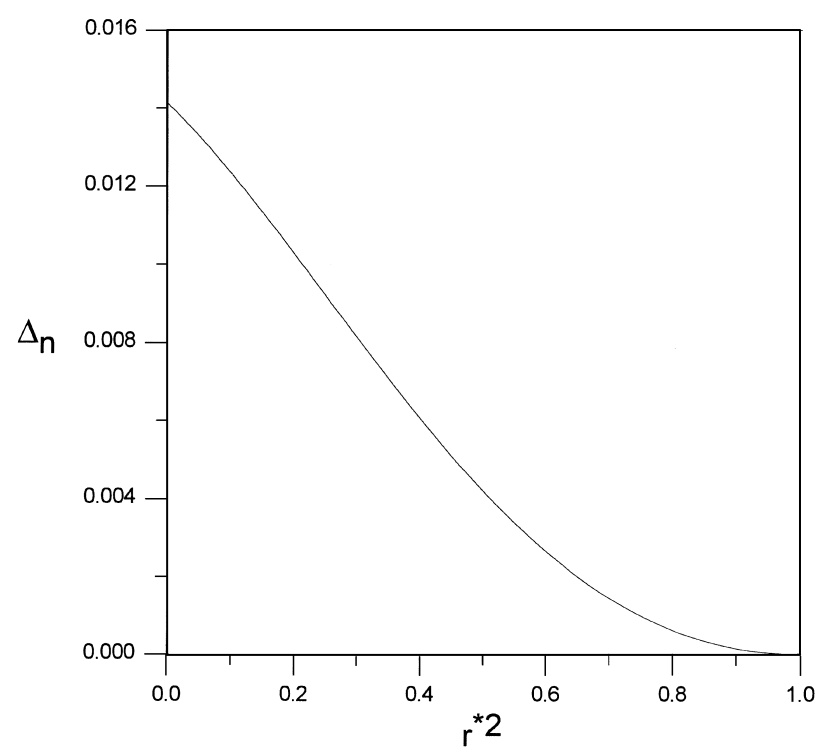

Fig. 9. Simulated variation of $\Delta n$ as a function of $r *^{2}$ for the case $R_{\mathrm{f}}^{*}=0.75$, $z_{\mathrm{BzMA}}^{*}=0.04$ and $z_{\mathrm{MMA}}^{*}=0.06$. Inner: $30 \%$ BzMA, $12 \% \mathrm{MMA}$, and $58 \%$ PMMA; outer: $40 \%$ MMA and 60\% PMMA.
According to Eqs. (8a) and (8b), this is equivalent to varying $D_{\mathrm{MMA}}$ at fixed $D_{\mathrm{BzMA}}$. As can be seen from Fig. 8, the larger the ratio $z_{\mathrm{MMA}}^{*} / z_{\mathrm{BzMA}}^{*}$, or $D_{\mathrm{MMA}} / D_{\mathrm{BzMA}}$, the lower the $\Delta n$ value, and the smaller the slope of the $\Delta n$ versus $r * 2$ curve near the center of a fiber. This result suggests that the RI distribution of the GRIN polymer fiber can be adjusted through the processing parameters of the coextrusion parameters.

A further test of the present mathematical modeling is shown in Fig. 9. By adjusting the composition of the reactants and the process parameters, a GRIN polymer fiber with about $70 \%$ of its region having a near parabolic RI distribution can be produced by the co-extrusion process examined.

\section{Conclusions}

A mathematical model was successfully developed to describe the RI distribution of the GRIN polymer fiber prepared by the closed co-extrusion process. An optimum value of the volume ratios of the inner/outer layer which cannot be too large or too small is required for obtaining a GRIN polymer fiber with an imaging quality. Also, the $\Delta n$ values increased with increasing initial concentration of the monomer BzMA in the reactant. The process parameters, such as monomer diffusion coefficients, fiber radius, extrusion velocity, and the diffusion length, show a significant effect on the RI distribution of the polymer fiber.

\section{Appendix A Nomenclature}

$\begin{array}{ll}A & \text { constant defined in Eq. (10) } \\ D_{r} & \text { diffusivity in the } r \text { direction } \\ D_{z} & \text { diffusivity in the } z \text { direction } \\ J_{n} & \text { Bessel functions of the first kind of order } n \\ L & \text { length of the diffusion zone } \\ n_{\mathrm{D}} & \text { RI } \\ r & \text { radial distance } \\ R & \text { radius of the diffusion zone } \\ R_{\mathrm{f}} & \text { radius of inner layer } \\ u & \text { extrusion velocity } \\ x & \text { mass fraction } \\ x_{\text {BzMAi }} & \text { initial mass fraction of BzMA in the inner layer } \\ x_{\text {MMAd }} & x_{\text {MMAo }}-x_{\text {MMAi }} \\ x_{\text {MMAi }} & \text { initial mass fraction of MMA in the inner layer } \\ x_{\text {MMAo }} & \text { initial mass fraction of MMA in the outer layer } \\ z & \text { distance measured from the top of the diffusion zone }\end{array}$

\section{Appendix A.1 Greek letters}

$\beta_{m} \quad$ positive root of $J_{1}\left(\beta_{m}\right)=0$

$\rho_{i} \quad$ density of component $i$

$\phi \quad$ parameter defined in Eq. (9a)

\section{Appendix A.2 Superscript}

$$
\text { dimensionless symbol }
$$




\section{References}

[1] Steele R, editor. Fourth International Conference on Plastic Optical Fibers \& Applications, Boston, MA, USA, Oct. 17-19, 1995. Information Gatekeeper, Inc.

[2] Koike Y, Harmer A, editors. Third International Conference on Plastic Optical Fibers \& Applications, Yokohama, Japan, Oct. 26-28, 1994

[3] Atkinson LG, Kindred DS, Zinter JB. Opt Photonics News 1994;5:28.

[4] Koike Y. In: Hornak LA, editor. Polymers for lightwave and integrated optics. New York: Marcel Dekker, 1992:71.

[5] Moore DT. Appl Opt 1980;19:1035.

[6] Ishigure T, Nihei E, Yamazaki S, Kobayashi K, Koike Y. Electro Lett 1995;31:467.

[7] Tagaya A, Koike Y, Nihei E, Teramoto S, Fujii K, Yamamoto T, Sasaki K. Appl Opt 1995;34:988.

[8] Yamamoto T, Mishina Y, Oda M, (Mitsubishi Rayon Co., Ltd). US Patent 4852 982, 1989.

[9] Mishina Y, Uotsu Y, Oda M, (Mitsubishi Rayon Co., Ltd). JP Patent 1-189602, 1989.

[10] Mishina Y, Uotsu Y, Oda M, (Mitsubishi Rayon Co., Ltd). JP Patent 2-16505, 1990.
[11] Mishina Y, Murata R, Uotsu Y, Oda M, (Mitsubishi Rayon Co., Ltd) JP Patent 2-33104, 1990.

[12] Tsai CC, Liu TJ, Chang YH, Tseng TW. Chem Eng Sci 1997;52:221.

[13] Perry GA, Witcher CE, (Peachtree Fiberoptics, Inc.). US Patent 5235660,1993

[14] Toyoda N, Mishina Y, Murata R, Uozu Y, Oda M, Ishimaru T, (Mitsubishi Rayon Co., Ltd). US Patent $5390274,1995$.

[15] Ho BC, Chen JH, Chen WC, Chang YH, Yang SY, Chen JJ, Tseng TW. Polym J 1995;27:310.

[16] Chen WC, Chen JH, Yang SY, Cherng JY, Chang YH, Ho BC. J Appl Polym Sci 1996;60:1379.

[17] Chen WC, Chen JH, Yang SY, Chen JJ, Chang YH, Ho BC, Tseng TW. In: Jenekhe SA, Wynne KJ, editors. Photonic and optoelectronic polymers, ACS Symposium Series, vol. 672. In press.

[18] Lorentz HA. Wied Ann Phys 1880;9:641.

[19] Lorenz LV. Wied Ann Phys 1880;11:70.

[20] Van Krevelen DW. In: Properties of polymers. 2nd ed. Amsterdam: Elsevier, 1976.

[21] Tong T-M, Ouano AC. Polym Eng Sci 1985;25:75-82. 